\title{
Implementing an Integrative Survivorship Program at a Comprehensive Cancer Center: A Multimodal Approach to Life After Cancer
}

\author{
Kathryn M. Glaser, PhD, Douglas C. McDaniel, DACM, LAc, ${ }^{2}$ Suzanne M. Hess, PhD, ${ }^{3}$ \\ Tessa Faye Flores, MD, ${ }^{4}$ Denise A. Rokitka, MD, $\mathrm{MPH}^{2}$ and Mary E. Reid, $\mathrm{PhD}^{4}$
}

\begin{abstract}
Background: This article describes the development of an integrative survivorship program at an urban National Cancer Institute-designated comprehensive cancer center with three closely linked components: a Survivorship Clinic with dedicated staff, a network of Support Services including Wellness, and an Integrative Medicine Program.

Development: We first defined the parameters of survivorship care and developed a patient-centric model that determined the optimal timeframes for transitioning these patients from the oncology clinic to a centralized survivorship clinic. Survivorship care includes the development of a survivorship care plan (SCP) for each patient at their initial visit to the program. Quality-of-life assessments are used in real time to guide clinical decision making to referrals to supportive care services, including educational events, expert consultations, and treatment using integrative and complementary therapies, access to legal services, community resource information, and support group activities for cancer survivors and caregivers. Integrative therapies were added to support the needs of this new program, including recruiting a nutritionist and acupuncturist, and developing a yoga, mindfulness, and Reiki program.

Population served: As of June 2018, 908 people have accessed our survivorship clinic, receiving a complete clinical assessment and SCP. Patients are routinely referred to support services based on the individual needs and ongoing symptoms from treatment. The majority of referrals are made to acupuncture, Healing Touch or Reiki, nutrition, psychosocial oncology, and yoga.

Conclusions: Developing a successful integrative survivorship program requires some essential features, including institutional support, strong leadership, a clear vision of how the clinical program will function, a dedicated team that is willing to do what it takes to get the program off the ground, and clinical oncology champions to refer patients into the program. With the development of this program, this multimodal approach to patient-centric care is maintained throughout the spectrum of care, from diagnosis to survivorship.
\end{abstract}

Keywords: cancer, survivorship care, integrative medicine, wellness

\section{Introduction}

M ANY ADVANCES HAVE BEEN MADE in survivorship care since the release of the Institute of Medicine's landmark report in 2006, "From Cancer Patient to Cancer Survivors: Lost in Transition." 1 The National Cancer Institute (NCI) defines cancer survivors as "anyone who remains alive and continues to function from the time of the diagnosis of cancer until the end of life." Many of the issues that plague patients under treatment persist after curative treatment is completed. It is estimated that $65 \%-80 \%$ of cancer survivors require additional physical and emotional support well beyond their treatment period. ${ }^{2}$ For the purposes of this discussion, survivorship care will be defined as addressing the post-treatment period.

In 2016, there were 7.4 million male and 8.2 million female survivors in the United States, and it is estimated there will be $>20$ million cancer survivors by $2026 .^{3}$ Although

Departments of ${ }^{1}$ Cancer Prevention and Control, ${ }^{2}$ Pediatrics, ${ }^{3}$ Research Support Services, and ${ }^{4}$ Medicine, Roswell Park Comprehensive Cancer Center, Buffalo, NY. 
standard-of-care guidelines for survivorship have been developed, ${ }^{2,4}$ there is a dearth of well-accepted models for implementing survivorship care into clinical settings. This article describes the development of a survivorship program at an urban NCI-designated comprehensive cancer center.

The Survivorship Program at Roswell Park Comprehensive Cancer Center (Roswell Park) was established in 2015 and was developed to aid patients and families in the transition from active treatment to rehabilitation and wellness. Our program was designed to bridge existing gaps in survivorship care, especially to address long-term symptom management and offer integrative therapies. The philosophy of the program is to provide support through patient and family-oriented care; to individualize a survivorship care plan (SCP) that will provide a map for the future; to maximize the level of health and wellness for every survivor and their support system through education, experiences, and therapies; and to improve the quality of life (QoL) for survivors and their support networks.

The program has three closely linked components. This includes a Survivorship Clinic with dedicated staff, a network of Support Services including Wellness, and an Integrative Medicine Program. This program was developed to provide patient- and family-centered care; viewing the whole person — body, mind, and spirit.

An initial needs assessment of Roswell Park cancer patients in November 2015, with just more than 1000 respondents, identified several unmet needs of cancer survivors and caregivers in three main areas: a strong desire for physical and psychosocial support during and after cancer treatment; a strong interest in nutrition education and physical activities, such as yoga; and co-ordinated support services to manage persistent symptoms from treatment. These results helped to focus the program development and to leverage support for creating new services. Although many resources existed at Roswell Park, they were not co-ordinated to meet the needs of cancer survivors and caregivers. In particular, there was no defined integrative medicine program.

A strategic business plan was developed to secure the financial and institutional support and infrastructure necessary for developing survivorship care. This included creating a survivorship clinic staffed by an internal and pediatric boarded physician, an oncology-trained nurse practitioner (NP), nurse clinical care co-ordinators, and patient care navigators to provide comprehensive physical and psychological assessments. Existing programs from Memorial Sloan Kettering Cancer Center and Mayo Clinic provided basic information on survivorship visit scheduling and reimbursement levels. Figure 1 shows the process of the program development and evaluation, which is described in greater detail in the following sections.

\section{Development: Defining Survivorship Care and Developing the Program}

During the initial planning phase for the survivorship program, the Director of Survivorship (M.R.) contacted existing survivorship programs to identify the models of survivorship care that were in use. The existing models were either centralized, where all patients are referred to a clinic and removed from primary oncology services, or disease

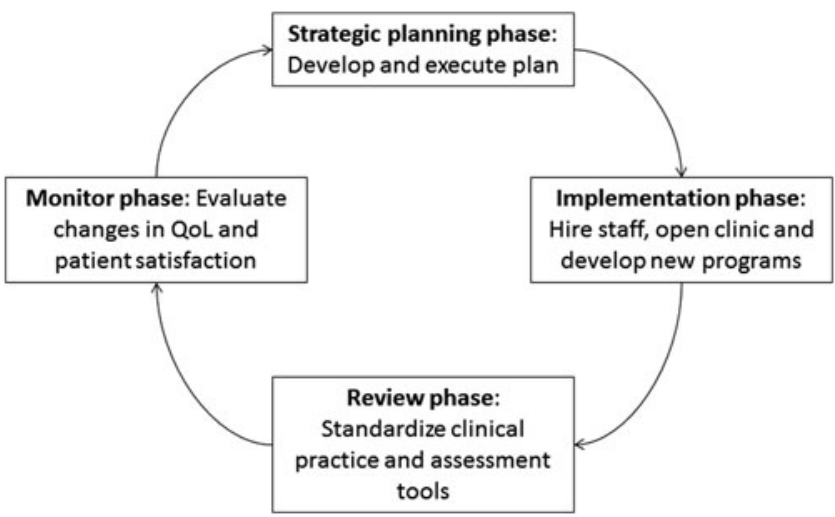

FIG. 1. Process of Survivorship Program Development. QoL, quality of life.

specific, where the survivorship support is embedded in the oncology clinics. Based on feedback from oncologists, an early determination was made that the decision to access the survivorship program lay in the hands of the oncology care teams and programs.

This led Roswell Park to adapt a mixed model where some services would transfer patients to the centralized survivorship program whereas others would prefer to provide survivorship care within the oncology practices (including the hematologic and urologic services). In addition, M.R. met with the other oncology disease sites to define a workable survivorship care pathway and to determine the optimal timeframe for transitioning these patients from the oncology service to the survivorship service. Referrals would not be mandated and the transition of patients, again, is based on the preferences of the specific oncology service.

There are three ways for patients to access the survivorship program. First, when patients complete treatment, a consult or one-time visit to survivorship provides the patient and family with a complete assessment and referrals at the start of the post-treatment period. This strategy gets the patient and family on track toward rehabilitation and wellness at the start of the recovery process. Documentation is provided back to the oncology team that can serve as the basis of an SCP.

The second avenue to survivorship is a transfer from oncology for long-term surveillance. The oncology team discusses this transition to survivorship with patients during a follow-up visit, and the transfer to survivorship providers is scheduled for the next follow-up visit. Again, a full assessment and referrals are provided, with treatment for symptoms and a surveillance plan (i.e., imaging) based on the preferences of the oncology team and National Comprehensive Cancer Network guidelines.

The final way to access survivorship is through selfreferral. Survivorship care should not be solely contingent on the preferences of the oncology team. Patients must be able to decide for themselves if they are ready for survivorship care. Regardless of how patients come into the program, close attention is paid to communicating with both the oncology team and the patient's community-based providers. This patient-centric model of survivorship care allows for time to address issues not directly related to cancer treatment; it streamlines access to multiple support services, 
special wellness programs, and integrative medicine. The equally clear benefit to the oncology practice is that there is more time to focus on patients who are newly diagnosed and undergoing active treatment.

To successfully implement the survivorship program, the transfer of patients from oncology clinics was required. The first step was to meet with the leaders of several disease sites (breast, urology, gastrointestinal [GI] including esophageal, gynecology, thoracic, lymphoma, and bone marrow transplant) to understand the standard course of survivorship surveillance, the demographics and numbers of patients surviving, and the sequelae of treatment-related symptoms normally seen in these populations. A critical step was identifying a physician champion from each disease site who was willing to refer patients and who supported the structure and goals of the program. The first disease site to take advantage of the survivorship program was breast oncology.

Given the large number of long-term ( $\geq 5$ years) breast cancer survivors coming in for annual appointments and early buy-in from the clinical team, this became a natural starting point. Many of these patients spent years seeing their oncologist: It was a delicate transition to make the patient feel supported and successful in beating their cancer and the importance of addressing their long-term survivorship and surveillance needs, without making them feel abandoned by their oncology team. The decision was made to have the provider introduce transferring to survivorship at an annual visit and if the patients agreed, the next annual appointment was scheduled in survivorship. Transfer is not mandatory but encouraged and provided a needed decompression of the clinic. In the 2 years that the survivorship clinic has been open, other disease sites have started to participate, predominantly gynecology, GI, and thoracic.

The Survivorship and Supportive Care Clinic opened in September 2016. Patients were slowly transitioned to survivorship over the first year. Operational funding was designated by the hospital to hire clinical staff, including a medical director, who serves as an oncogeneralist to provide clinical oversight and to develop the clinical protocols for surveillance; an oncology-trained NP; a nurse clinical care co-ordinator; and a patient care navigator. New clinical protocols have been activated, including targeted screening for cardiotoxicities, cognitive deficits, nutritional problems, and second primary tumors.

In addition, the clinical space provides a central hub for existing supportive services, including psychotherapy, psychology, social work, nutrition, and wellness services. A new Director of Rehabilitation and Wellness was hired to work closely with the program to provide access to a stateof-the art center for physical and occupation rehabilitation and lymphedema therapy. Donation funds were raised in February 2017, and an Integrative Medicine Program Manager was hired to develop and provide a vibrant program of acupuncture and other integrative modalities. Finally, through a donation-driven grant program for QoL improvements, continual support is available for integrative health programs, including Healing Touch (HT), massage therapy, support groups, yoga, mindfulness, and education sessions focused on wellness. Again, the survivorship program structure and services were driven by the needs expressed by our survivors and their families.

SCPs are an established part of survivorship care. The clinical team is committed to providing every patient seen in survivorship with a care plan, regardless of the time since diagnosis. This personalized care plan, designed to incorporate the suggested components from the American Society of Clinical Oncology, maps surveillance schedules, potential long-term problems related to treatment, cancer screening, and support service referrals. The plan also provides clear recommendations for the role that primary care providers (PCPs) can plan in the patient's long-term care, thus actively re-engaging the PCP in survivorship care. This plan is given to the patient and a copy is sent to the community providers.

Although there are mandated requirements for SCPs completion, these requirements do not address the quality of survivorship care. They exclude groups of survivors (such as stage IV patients) and have led to a diversion of funds that shift the focus away from survivorship care. Although many institutions, such as Roswell Park, strive to meet these requirements, it is not at the expense of providing highquality, comprehensive survivorship care to all of our cancer survivors and their caregivers.

At the initial appointment and then repeated annually, the European Organization for Research and Treatment of Cancer (EORTC) quality-of-life questionnaire (QLQ) is administered to each patient. This questionnaire incorporates five functional scales (physical, role, cognitive, emotional, and social) and three symptom scales (fatigue, pain, and nausea, and vomiting). In addition, it includes a global health status and a number of single items assessing common symptoms reported by cancer patients (dyspnea, loss of appetite, insomnia, constipation, and diarrhea), as well as perceived financial impact of the disease.

Clinical thresholds for the physical functioning $(<80)$, emotional functioning $(<70)$, fatigue $(>39)$, pain $(>25)$, and role function $(<90)$ scales of the EORTC QLQ-C30 are based on literature that supports using these thresholds to identify clinically important problems that should be discussed with the patient. ${ }^{5}$ The results are used in daily clinical practice to guide clinical decision making to referrals to supportive care services, including educational events, expert consultations, and treatment using integrative and complementary therapies, access to legal services, community resource information, and support group activities for cancer survivors and caregivers.

\section{Population Served}

As of June 2018, 908 people have accessed our survivorship clinic. The disease sites that have transferred the most patients include breast oncology (62\%), gynecologic oncology (23\%), and pediatric oncology (11\%). Based on the QLQ summary scores, patients are routinely referred to psychosocial oncology, physical and occupational therapy, nutrition, integrative medicine, and wellness programs to help meet their ongoing needs related to their recovery. The majority of referrals are made to acupuncture, HT, nutrition, psychosocial oncology, and yoga. In June 2018, a dedicated nutritionist was hired to offer consultations to this unique population and a second acupuncturist was hired to expand our acupuncture offerings to a waiting list of more than 100 patients. In September 2018, a Bio-field Therapies Program encompassing HT and Reiki was implemented to meet the expanding needs of energy-based therapies.

We have found that many survivors lack the information and understanding to adequately safeguard their continued 
recovery and support their overall return to health and wellbeing to prevent recurrence or occurrence of secondary cancers. In an effort to reconnect with patients who are currently not receiving any survivorship care, and to connect with those treated in other cancer centers that do not have a survivorship program, we are currently in the process of doing outreach to both of these groups to provide information on what the program has to offer them and the importance of survivorship care in general.

For the young adult (YA) cancer survivors, a special mailing was developed to identify and promote the new survivorship program. Patients diagnosed between the ages of 18 and 37 years and seen for follow-up at the cancer center between January 2016 and February 2018 were identified. Mailings were staggered, and a total of 2148 letters were mailed out from January 2017 to February 2018. Of this number, only seven patients responded to initiate a YA survivorship appointment.

This low response rate was disappointing, but it may be due to a number of factors unique to the YA population. Direct mail may not be the best way to communicate with patients in this age demographic. Using email, text, or other forms of social media to communicate information regarding survivorship may have elicited a larger response since social media platforms are the major form of communication used by this age group. Also, younger people tend to move more frequently, leading to potentially outdated mailing addresses.

The 18- to 37-year-old group is a broad range that encompasses survivors in many different stages of life presenting unique challenges to connecting and communicating with this diverse population. Many YAs experience fewer late or lingering effects of their treatment compared with older populations. They are more likely to be in school, starting careers, getting married, and potentially having families. They may not understand the importance of regular survivorship care to help maintain their general health and well-being, as well as prevent recurrence and screen for secondary cancers.

However, others are plagued with late or lingering physical and emotional effects of their treatment, making it difficult for them to restart their lives and move forward. This group may still need family support for housing, finances, and transportation. They may also continue to be overwhelmed with medical oncology appointments, making any additional appointments too stressful.

To reach this population effectively, communication should be patient centered and individualized to meet the needs of the YA. Survivorship and integrative care should focus on health education and peer support that fosters independence. YAs are a unique and challenging group with diverse needs throughout their survivorship journey. Listening to their needs and tailoring programs around their specific challenges will help them feel supported and engaged to make an investment in their future health and well-being.

\section{Integrative Medicine}

Since the inception of the Integrative Medicine program in March 2017, acupuncture and other integrative modalities have been available through the survivorship clinic to help manage late and lingering effects of treatment, preserve and promote fertility, and support general health and well-being. Services are provided in the survivorship clinic by appointment and are offered free of charge to all patients. For those survivors on active treatment, services are available during chemo infusion or inpatient stay. To date, more than 900 acupuncture treatments have been delivered to pediatric, adolescent and young adult (AYA), and adult survivors through our survivorship clinic.

From the start, interest was high in the adult survivorship program in receiving acupuncture services with patient selfreferral and referrals from the Palliative Care and Pain Management teams, especially in light of the ongoing opioid crisis. As the demand for in-house acupuncture grew, we realized we would not be able to provide the necessary resources to meet the demand. This prompted the development of survivorship specific continuing education training for local acupuncturists interested in treating cancer survivors. This, in turn, provided us with a referral network for patients who could not routinely come to our clinic for acupuncture. It also provided Roswell Park as a resource to those community acupuncturists should any questions related to the patients' medical condition arise.

The program began with one fulltime acupuncturist focused on treating pediatric and AYA patients and survivors based on funding sources. It soon became evident that there was great interest and need for acupuncture in the adult survivorship population, and additional funding was secured to offer acupuncture services two afternoons per week to our adult survivors. We continue to look for community partners to provide space and capacity to expand our services even further. Disease-specific continuing education is currently being developed for both primary care physicians and acupuncturists to further broaden the network of community providers with the necessary competencies to treat cancer survivors.

As opportunities arise to provide comprehensive acupuncture care throughout the hospital in adult chemo infusion, radiation oncology, and expanded inpatient coverage, the ability to meet these needs with our current model is already being challenged. Maximizing our limited resources by possibly moving from individualized outpatient treatments to group treatments for specific high-volume symptoms (i.e., neuropathy or specific disease groups) may help to alleviate the capacity issue. Collecting and reporting data on patient utilization, reported outcomes, and reduction of costs may help influence the inclusion of acupuncture and other integrative services in health care coverage, offsetting the cost and allowing for an expansion of the program.

Institutional support, strong leadership, and clinical champions have allowed the Integrative Medicine program to move forward with the clear and evolving mission of making acupuncture and other integrative offerings available to all patients treated at Roswell Park. To fulfill this vision, it is necessary for the program to be supported by donor funding. This presents a unique challenge that as demand for services increases, the ability to provide those services is limited unless funding is secured to sustain the existing program and support growth to hire additional providers. This highlights the importance of strong business planning and donor support to continue to offer these integrative therapies in a timely fashion.

\section{Healing Touch}

HT is a noninvasive, complementary, holistic bio-field therapy offered twice a week, $3 \mathrm{~h}$ each day, for 30-min sessions in chairs, in an annex to the survivorship waiting 
Table 1. Results from Yoga and Healing TOUCH PROGRAMS

\begin{tabular}{|c|c|c|}
\hline Program & Measure & Total \\
\hline $\begin{array}{c}\text { Yoga Program } \\
\text { (May 2017- } \\
\text { June 2018) }\end{array}$ & $\begin{array}{l}\text { Total participants } \\
\text { Total sessions held } \\
\text { Survivorship patients } \\
\text { Caregivers } \\
\text { Survivorship staff } \\
\text { Female } \\
\text { Male } \\
\text { Average age } \\
\text { Range }\end{array}$ & $\begin{array}{l}59 \\
58 \\
56 \% \\
25 \% \\
19 \% \\
92 \% \\
8 \% \\
47 \\
17-72 \\
\text { years old }\end{array}$ \\
\hline $\begin{array}{l}\text { Healing Touch } \\
\text { Program } \\
\text { (March 2017- } \\
\text { March 2018) }\end{array}$ & $\begin{array}{l}\text { Total participants } \\
\text { Total sessions held } \\
\text { Total 30-min Healing } \\
\text { Touch Sessions Given } \\
\text { Active patients (surgery, } \\
\text { chemo, radiation) } \\
\text { Cancer survivors } \\
\text { Caregivers } \\
\text { Staff } \\
\text { Others (screening, pain, } \\
\text { palliative) } \\
\text { Female } \\
\text { Male } \\
\text { Age range }\end{array}$ & $\begin{array}{c}360 \\
97 \\
760 \\
\\
22 \% \\
44 \% \\
35 \% \\
15 \% \\
7 \% \\
\\
85 \% \\
15 \% \\
14-93 \\
\text { years old }\end{array}$ \\
\hline
\end{tabular}

room starting in March 2017. The program is led by a Healing Beyond Borders certified HT practitioner through a hospital-based HT volunteer program. On average, 6 to $10 \mathrm{HT}$ Level I trained volunteers donate their time each week. In the first year of the program, more than 750 thirty-min chair sessions were given by more than 14 different Level I trained HT volunteers (Table 1). To date, more than 490 individual patients, caregivers, and staff have enjoyed $\sim 1200$ sessions. In September 2018, the program expanded to offer 1 day of Reiki. The location makes it easy for clinicians to refer and patients to take advantage either before or after other scheduled survivorship appointments.

\section{Yoga}

Since May 2017, 1-h restorative yoga classes have been offered in 8-week cycles. Program evaluations were conducted at the end of each session between April and June 2018 to evaluate the effectiveness of the program and participant satisfaction. Sessions increased to biweekly in May 2018 based on evaluations and demand. Participants include cancer survivors who were medically cleared and completed treatment at least 2 months prior, caregivers, and survivorship staff. To date, 58 classes have been held (56\% survivors, 25\% caregivers, and 19\% survivorship staff) (Table 1). Initial data suggest that yoga is a feasible and effective intervention for cancer survivors and caregivers with high participant satisfaction from all respondents rating the program and instructors good to excellent.

\section{Oncology Massage}

Beginning January 2017, oncology massage therapists were identified to provide services for patients and caregivers to promote relaxation, reduce anxiety, and improve sleep. From January 2017 to June 2018, 10-min chair massages were offered to patients, caregivers, staff, and volunteers, with first priority given to patients. Sessions lasted $4 \mathrm{~h}$ with two massage therapists per session. Initially offered twice a month, it was expanded to four times a month in May 2018. There is an average of 34 massages per shift, and since initiating the program, 1080 individuals have received massage $(70 \%$ patients, $20 \%$ caregivers, and $10 \%$ staff and volunteers). Massage is offered on site in two locations, the Patient Resource Center and Survivorship Clinic. Massage dates and times are listed in the patient activities newsletter, fliers are placed in waiting areas and lobbies, and invitations are hand delivered to patients and families on the day of the massages.

\section{Conclusions}

Developing a successful integrative survivorship program requires some essential features. This includes institutional support, strong leadership, a clear vision of how the program will function, a dedicated team that is willing to do what it takes to get the program off the ground, and clinical champions. Continued funding for integrative medicine programs to meet the demands and sustain these programs is also a key to success.

When discussing the benefits of survivor-centered care with clinical teams, no one disagrees with the concept because it makes sense, supporting both the patients and the clinics in optimizing ongoing care. Hesitancy only comes from not knowing how to make the transition into survivorship smooth and seamless for the patient and the oncologist. To make the program truly successful, it needs to be easy for patients to access the program and for clinicians to transfer the patients with the confidence that the surveillance will be at a high standard and patients will have access to integrative therapies to address long-term symptom burden.

Having a clear vision for the program that incorporates the mission of both survivorship care and the organization delivering care is essential to program success. The key is to remain focused on the goals of the program: to deliver patient- and family-centered care and to personalize each care plan so that each survivor feels empowered and special. With the development of this survivorship program, this multimodal approach to patient-centric care is maintained throughout the spectrum of care, from diagnosis to survivorship.

\section{Acknowledgments}

This work was supported by Roswell Park Comprehensive Cancer Center and NCI grant P30CS016056. The Integrative Medicine program is supported by the Roswell Park Alliance Foundation. The authors wish to acknowledge and thank the Roswell Park Healing Touch volunteers and all other volunteers who donated their time in the Supportive Care Clinic. 


\section{Author Disclosure Statement}

No competing financial interests exist.

\section{References}

1. Institute of Medicine and National Research Council. From Cancer Patient to Cancer Survivor: Lost in Transition: An American Society of Clinical Oncology and Institute of Medicine Symposium. Washington, DC: The National Academies Press, 2006.

2. National Comprehensive Cancer Network. Survivorship (Version 1.2018). Online document at: https://www.ncen .org/professionals/physician_gls/default.aspx\#supportive, accessed August 22, 2018.

3. Miller KD, Siegel RL, Lin CC, et al. Cancer treatment and survivorship statistics, 2016. CA Cancer J Clin 2016;66: 271-289.
4. American Society of Clinical Oncology. Guidelines on Survivorship Care. Online document at: https://www.asco .org/practice-guidelines/cancer-care-initiatives/preventionsurvivorship/survivorship-compendium-0, accessed January 23, 2018.

5. Giesinger JM, Kuijpers W, Young T, et al. Thresholds for clinical importance for four key domains of the EORTC QLQ-C30: Physical functioning, emotional functioning, fatigue and pain. Health Qual Life Outcomes 2016;14:87.

Address correspondence to: Kathryn M. Glaser, PhD Department of Cancer Prevention and Control Roswell Park Comprehensive Cancer Center Elm and Carlton Streets Buffalo, NY 14263

E-mail: kathryn.glaser@roswellpark.org 